\title{
A Double Edged Sword-anticoagulation Treatment in a Patient with Mechanical Prosthetic Mitral Valve Complicated by Brain Hemorrhage
}

\section{Noam Fink $^{1 *}$, Ashraf Hamdan ${ }^{1}$, Ori Vatury ${ }^{1}$, Orly Goitein ${ }^{2}$ and Sagit Ben Zekry ${ }^{1}$}

${ }^{1}$ Non Invasive Cardiology Unit, Leviev Heart Institute, Sheba Medical Center and Sackler School of Medicine, Tel Aviv University, Israel ${ }^{2}$ Diagnostic Imaging, Leviev Heart Institute, Sheba Medical Center and Sackler School of Medicine, Tel Aviv University, Israel

\begin{abstract}
In the current case presentation, a patient with prosthetic mechanical mitral valve developed brain hemorrhage following head trauma. Cessation of anticoagulation treatment resulted in left atrial thrombus; patient presented with recurrent emboli events to major arteries that required recurrent embolectomy. This case highlights the clinical conflict of restarting vitamin $\mathrm{K}$ anticoagulation following brain hemorrhage in a high risk patient and demonstrates the importance of multimodalities imaging in assessing those patients.
\end{abstract}

Keywords: Intracranial hemorrhage; Mechanical valve; Anticoagulation

\section{Case Report}

A 74-year-old female patient was admitted with a syncopal episode and head trauma. Head CT scan revealed traumatic frontal subarachnoid hematoma (Figure 1A). The medical history was significant for paroxysmal atrial fibrillation and mitral valve replacement with a mechanical valve 10 years ago. The INR on admission was 3.1 and patient was treated conservatively. The warfarin was held and her INR returned to normal values spontaneously with no coagulation factors replacement. Fourteen days later (during which she did not receive anticoagulation), a follow up head CT showed partial resolution of the hematoma and warfarin was renewed without any bridging therapy. A day later, the patient was re-admitted due to bilateral leg weakness and the physical examination was consistent with bilateral leg ischemia. CT angiography revealed thrombotic obstruction of the distal aorta proximal to the bifurcation to the ileac arteries (saddle thrombus), (Figure 1B) and the patients underwent urgent bilateral trans-femoral thrombectomy with full clinical resolution. A trans-esophageal echocardiography revealed a normal functioning mechanical mitral valve with a large mobile thrombus $(1.2 \times 1.2 \times 1.4$ $\mathrm{cm}$ ) in the left atrium (LA). The thrombus bounced of the mechanical
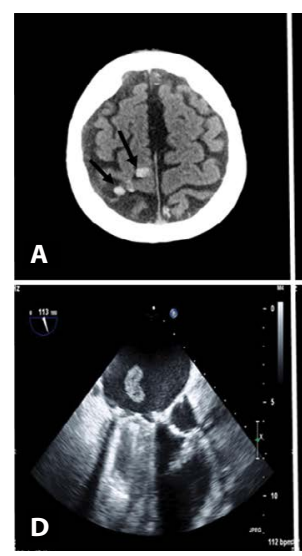
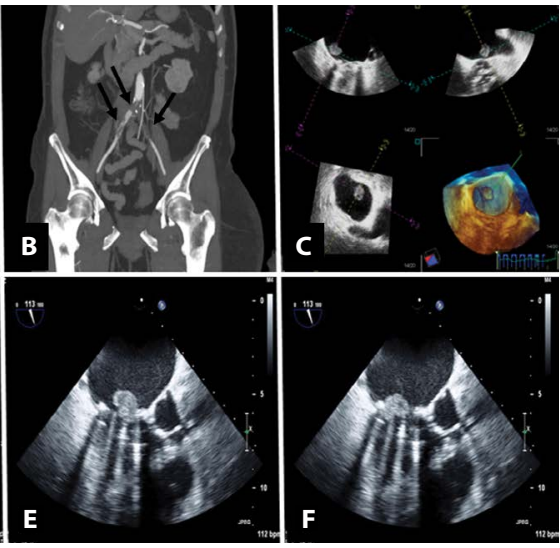

Figure 1: A-CT scan of the brain demonstrating subarachnoid hematoma (arrows head); B-CT angiography demonstrating the obstruction of the distal aorta, proximal to the bifurcation to the ileac arteries; C-LA thrombus visualized by 3 -deminsional transesophageal echocardiography; D-F-The mobile LA thrombus is bounced of the mechanical mitral valve.

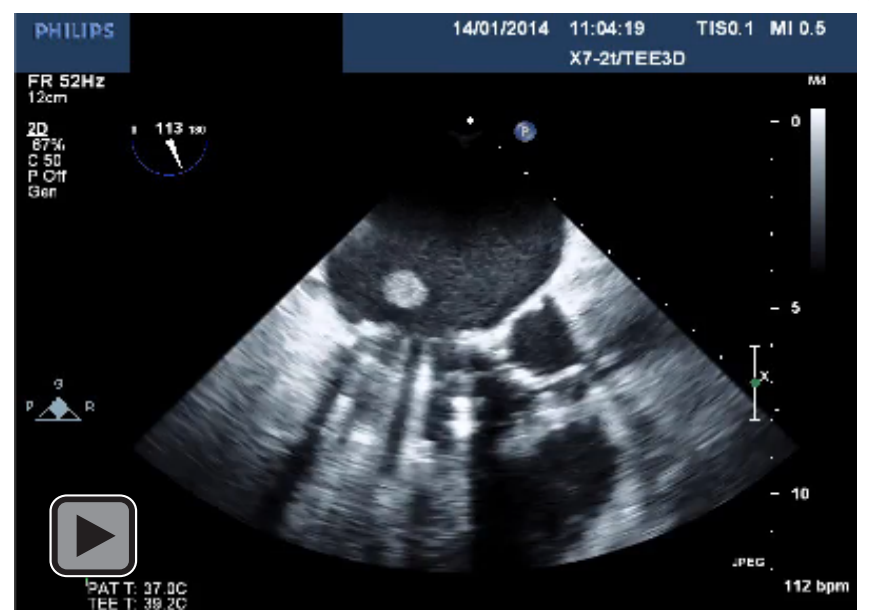

Video 1: 2D - Two dimensional transesophageal echocardiography of the mobile thrombus.

valve intermittently (Figures 1C-F) (Videos 1 and 2). Cardiothoracic surgeons neglected open heart surgery due to the recent head trauma and hemorrhage. Consequently, patient was treated with warfarin and enoxaparin until reaching an INR of 3 . There was no resolution of the LA thrombus on serial echocardiography but since surgery was not possible, she was discharged. The following day, the patient presented with acute ischemia of the right arm; Ultrasound Doppler confirmed right axillary artery obstruction and the patient underwent right brachial embolectomy. Her INR on admission was 2.7. At this time, the echoacardiography showed disappearance of her LA thrombus. Patient was discharged uneventfully and she is doing well.

*Corresponding author: Noam Fink, Leviev Heart Center, Sheba Medical Center Tel Aviv University, Israel, Tel: +1972529242464; Fax: +197235305789; E-mail: noamfink@bezeqint.net

Received April 26, 2016; Accepted May 25, 2016; Published May 30, 2016

Citation: Fink N, Hamdan A, Vatury O, Goitein O, Zekry SB (2016) A Double Edged Sword-anticoagulation Treatment in a Patient with Mechanical Prosthetic Mitral Valve Complicated by Brain Hemorrhage. J Cardiovasc Dis Diagn S1: 007. doi:10.4172/2329-9517.S1-007

Copyright: (c) 2016 Fink N, et al. This is an open-access article distributed under the terms of the Creative Commons Attribution License, which permits unrestricted use, distribution, and reproduction in any medium, provided the original author and source are credited. 
Citation: Fink N, Hamdan A, Vatury O, Goitein O, Zekry SB (2016) A Double Edged Sword-anticoagulation Treatment in a Patient with Mechanical Prosthetic Mitral Valve Complicated by Brain Hemorrhage. J Cardiovasc Dis Diagn S1: 007. doi:10.4172/2329-9517.S1-007

Page 2 of 2

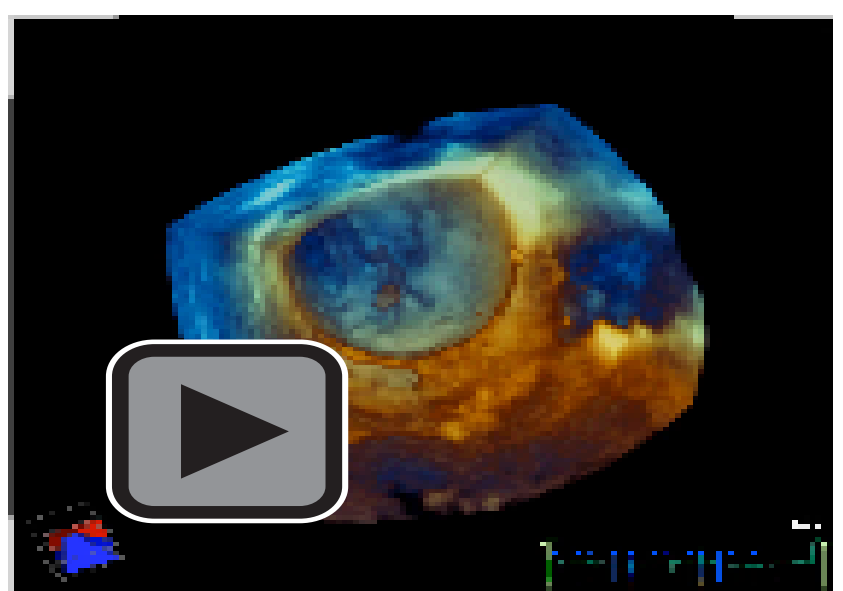

Video 2: 3D - Three dimensional reconstruction of the mobile thrombus.
This case highlights the conflict of restarting vitamin $\mathrm{K}$ anticoagulation (VKA) following brain hemorrhage. The European Stroke Initiative Writing Committee Suggest reinstituting VKA not earlier than 14 days following intracranial bleeding in patients at a high thromboembolic risk [1] while the AHA guidelines do not provide a specific time frame [2]. Moreover, none of the guidelines discussed the dilemma of restarting VKA simultaneously with heparin or low molecular weight heparin as the literature is limited. In a patient such as the one presented, with a high risk for thromboembolic events (presence of mechanical prosthetic mitral valve with paroxysmal atrial fibrillation) and small subarachenoid hematoma that is well controlled, we might consider earlier initiation of VKA. Administration of heparin or low molecular weight heparin until the INR is in the therapeutic range should also be considered.

\section{References}

1. Steiner T, Salman RAS, Beer R, Christensen H, Cordonnier C, et al. (2014) European stroke organization (ESO) guidelines for the management of spontaneous intracerebral hemorrhage. Int. J Stroke 97: 840-855.

2. Hemphill JC, Greenberg SM, Anderson CS, Becker K, Bendok BR, et al. (2015) Guidelines for the management of spontaneous intracerebral hemorrhage: a guideline for healthcare professionals from the American Heart Association/ American Stroke Association. Stroke 46: 2032-2060. 